\title{
Patients as team members: opportunities, challenges and paradoxes of including patients in multi-professional health-care teams
}

\author{
Graham P. Martin \& Rachael Finn
}

\begin{abstract}
Current health-care policy emphasises the need for more collaborative, team-based approaches to providing care, and for a greater voice for service users in the management and delivery of care. Increasingly, policy encourages 'partnerships' between users and professionals so that users too effectively become team members. In examining this phenomenon, this paper draws on insights from the organisational-sociological literature on team work, which highlights the challenges of bringing together diverse professional groups but which has not to date been applied in contexts where users, too, are included in teams. Using data from a qualitative study of five pilot cancergenetics projects, in which service users were included in teams responsible for managing and developing new services, it highlights the difficulties involved in making teams of such heterogeneous members-and the paradoxes that arise when this task is achieved. It reveals how the tension between integration and specialisation of team members, highlighted in the literature on teams in general, is especially acute for service users, the distinctiveness of whose contribution is more fragile, and open to blurring.
\end{abstract}

Published in

Sociology of Health \& IIIness 33(7): 1050-1065

http://www.blackwellpublishing.com/shil_enhanced/

http://onlinelibrary.wiley.com/doi/10.1111/j.1467-9566.2011.01356.x/abstract

doi: 10.1111/j.1467-9566.2011.01356.x

\section{Introduction}

Various reforms to the organisation and delivery of health services have sought to improve the quality, safety and patient-centredness of health-care provision. Policymakers have placed particular emphasis on breaking down the barriers between the professional groups involved in delivering health care, and between the providers and users of health services. Both these sets of reforms - the creation of multidisciplinary teams, and the involvement of service users such as patients and carers - are seen as a means of improving collaboration, knowledge sharing and accountability in health care, reducing fragmentation in the way care is delivered, improving quality and ensuring that services are built around patients' needs and wishes.

Increasingly, service-user involvement in health care is itself expected to take a more collaborative form, in order to overcome the power inequalities that are perpetuated when users are merely 'consulted' over service provision (Charles and DeMaio 1993). Service-user involvement is to take forms such as partnerships, which seek to emulate the equality of influence and close, cross-professional working of the multidisciplinary team. The literature on user involvement in health care and other fields of public services has documented mixed success in such efforts (Barnes et al. 2007, Harrison and Mort 1998, Martin 2008b). To date, however, it has 
not drawn on organisational sociological writing on team work in seeking to illuminate and understand these successes and failures.

In this paper, we seek to utilise this rich literature to offer new insights into the practice of service-user involvement. Using data from a study of five pilot projects in the British National Health Service (NHS) where practitioners from various professions were brought together with users to form management teams, we note how key factors identified by this literature facilitated or obstructed team integration and equality of influence. We extend these insights in relation to the particular situation facing service users-as 'outsiders' from beyond the professional norms and organisational customs of the health service. We also illustrate how the purpose of the team - as a means of reconciling the integration and specialisation of different roles-created particular challenges for service users, with important implications for how policy should determine the role of service-user involvement, cultivate its influence, and define its effectiveness.

Firstly, however, we review the literatures on team work and service-user involvement in more detail.

\section{Collaboration between and beyond professions}

Across developed-world health services, collaboration at various levels has been advocated by policymakers and academics for some time. In the NHS, policy has mandated collaborative networks between organisations at the macro level (Martin et al., 2009), integrated services at the meso level (Glendinning 2003), and cross-professional teams including members from diverse backgrounds at the micro level (Cott 1998). Micro-level multidisciplinary teams, integrating the contributions of diverse professionals towards a common clinical or organisational aim, are claimed to improve the quality of provision by offering more patient-centred services, organised around the needs of individuals rather than the boundaries of the professional or organisational system (Opie 1997). In particular, they are seen as a key means of reconciling two opposing imperatives in the delivery of health care: the need for integration and the need for specialisation in an increasingly fragmented division of labour (Donnellon 1996). Effective integration involves the creation of interdependent relationships across professional boundaries, so that the diverse bodies of expertise held by different groups are shared effectively and deployed synergistically (Donnellon 1996, Dingwall 1980).

Consequently, teams, partnerships and other forms of cross-professional working have been endorsed extensively in the policy literature. In the UK, the ability to work in teams is deemed a crucial characteristic of the modern health professional (e.g. General Medical Council 2006). Lack of team work is seen as a risk to patient safety, found by the Bristol Royal Infirmary (BRI) Inquiry (2001 p.276) to have created "tribal barriers" which gave rise to protracted deficiencies in paediatric heart surgery. Subsequent government policy has similarly emphasised the centrality of multi-professional collaboration to NHS reforms. The NHS Next Stage Review notes progress in instituting teams as key organisational units in the NHS- "Conversations about quality take placed in multi-disciplinary teams rather than in corridors"-but calls for further progress: "For patients, the team must go beyond individual organisations - they expect everyone in the NHS [...] to work together, to give them [...] high quality, integrated care" (Secretary of State for Health 2008).

Teams and other collaborative working practices, then, seem a solution to many of the challenges facing health care, from efficiency to safety to patient experience. However, studies of teams in practice find a more mixed picture, with various empirical studies illustrating the problems and challenges that accompany team work. This sociological literature highlights how contradictory views about the nature and purposes of teams-such that "all groupings of practitioners can interpret the phenomena as in their best interests"-give rise to conflict as the team is put into practice (Lotz and Kristensen 2009 p.9). From discourse-analytic perspectives, Griffiths (1997), Finn (2008) and Learmonth (2009) have highlighted how the term 'team work' acts as a "loose rubric rather than a detailed template for action" (Griffiths 1997 p.60), which 
may be constructed in very different ways in practice, and which therefore lends itself to strategic action by those who become 'team members'. Finn (2008) finds that this results in a tendency towards reproduction of dominant power relationships, such that the potential for greater openness, knowledge exchange and learning-and thus safer, more effective practice-is negated. Besides these discourse-analytic perspectives, various authors find that multidisciplinary working frequently fails to mitigate boundaries between professions (Cott 1998, Opie 1997), especially in relation to lower-status staff outside the clinical professions (Finn et al., 2010a). As a result, teams and other micro-level collaborative organisational forms often tend to exacerbate the fragmentation of professional groups, rather than facilitating their integration (Dingwall 1980, Donnellon 1996, Finn, 2008, Griffiths 1997), though local contexts can mitigate this (Finn et al., 2010b, Mueller et al. 2000, Theberge 2008).

Similarly, rather than creating 'flatter', more egalitarian working practices, team working often reproduces existing hierarchies of power and control (Finn 2008). To displace control as a means of regulating working practices requires the development of trust (Das and Teng 2001, cf. Donnellon 1996 pp.18-19), as a means of dealing with uncertainty and ensuring that those involved in co-operative work behave appropriately. Trust - in the sense of the "expectations held by one party that another will behave reliably and predictably" (Tomlinson 2005 p.1172) — can take a number of forms (Das and Teng 2001, Newell and Swan 2000), which can substitute for control in providing a basis for collaborative working. Trust may have various foundations, from initial knowledge of the other party's reputation to the trust found in long-term reciprocal relationships, but the more resilient and robust forms of trust take considerable time to develop (Ring and van de Ven 1994). Consequently, teams and other micro-level collaborative organisational forms frequently rely on more conventional, hierarchical mechanisms of control rather than on fragile and difficult-to-develop trust (Tomlinson 2005, Walker et al. 2007), though again, there are exceptions to this rule (Finn et al., 2010b).

Policy on the involvement of service users in the management of public services has developed alongside that on inter-professional collaboration, and, at least in part, with a view to addressing many of the same perceived deficiencies in public-service delivery, such as effectiveness and user-centredness (Abelson et al. 2003). In health care, from previous marginality, the involvement of service users is now de rigueur in the NHS and many other systems. Much of the policy and associated literature advocating multidisciplinary collaboration also demands greater service-user involvement as a parallel means of improving health care (e.g. Department of Health 2003). Alongside its calls for a transformation of the "tribal" culture of health professionalism, for example, the BRI Inquiry (2001 p.409) saw involvement as a key means of creating a safety culture within the NHS: "involvement of patients and the public must be embedded in the structures of the NHS and permeate all aspects of healthcare."

Not only are the aims of user involvement similar to those of policy around team work, but increasingly, the forms it should take are the same. In the British context, the Next Stage Review demands that the NHS locally "forge a new partnership between professionals, patients and their carers" (Secretary of State for Health 2008 p.20). Users are thus increasingly included in collaborative forums and teams where knowledge is exchanged to improve services. Thus the concept of the multidisciplinary team is being expanded to include patients and carers as well, as user involvement comes to involve not just consultation, but partnership, with shared decision making, shared responsibility and some degree of equality of influence between professionals and users (Chadderton 1995, Charles and DeMaio 1993, Rutter et al. 2004).

Given the challenges already noted of making functional, trusting teams out of different professional groups with diverse cultures, norms and interests, it is to be expected that similar problems will arise when a group altogether foreign to health-service culture-service users-is added to the mix. The literature on public participation and service-user involvement is largely circumspect about the prospects for impact of users' views on public-service governance (Barnes et al. 2007, Harrison and Mort 1998, Martin, 2008b). There are several recurrent themes in this 
literature. The institutions that govern health-service professionals often act to deter them from engaging fully with users, encouraging professionals to construct users less as knowledgeable partners and more as lay patients (Martin 2008b, Barnes et al. 2007): despite the apparent elevation of users to the status of legitimate stakeholders in health-service governance, in practice their views often remain denigrated. Health-care professionals tend to be vested with control over the processes by which service users are consulted and how their input is used, and exercise this power in choosing which aspects of service-user involvement to take up and which to quietly discard (Harrison and Mort 1998, Milewa et al. 1999). And the way in which user-involvement processes are set up often reinforces these divisions between staff and service users, with consultation often preferred over partnership, and a consequent arms-length relationship between service users and staff (Martin 2008b). These features of public-participation and user-involvement processes are apparent in approaches adopted worldwide, for example Canada's regional and community-level health organisations (Contandriopoulos 2004) and efforts in the United States to increase public participation in health policy and planning (Sleath and Rucker 2001).

Given these longstanding problems with user involvement, the task of effectively including users in the multidisciplinary team seems highly challenging. Yet for all its challenges, the notion of expanding the team to include the user does at least offer the possibility of engendering a more co-operative, less hierarchical relationship by promoting collaboration and parity between users and professionals - even if it does not always achieve this among staff. How far, then, might the integration of users into teams which seek to foster collaboration and knowledge exchange achieve these aims? How can a grounding in the sociological literature on teams, previously not applied to the study of user involvement, enrich our understanding of this process? Can the insights gained from this perspective offer any suggestions about how the challenges in gaining influence for user involvement might be mitigated? It is these questions of process (rather than the rather different question of the outcome of incorporating users into teams) that this paper addresses.

\section{Setting and methods}

The empirical work on which this paper draws is a comparative study of seven pilot NHS cancergenetics services, each of which was mandated (and sought in different ways) to foster a 'partnership' with service users in the development and management of their projects. This was as part of a more general mandate to foster collaborative working between cancer specialists, genetics specialists and other groups in the health service (such as primary-care doctors and nurses) to create more 'joined-up' cancer-genetics services that would better meet patients' needs (exactly who should be involved in any given team was left open to pilots to define). Service-user involvement, then, was part of a wider emphasis on collaboration, premised on the expectation that more effective, joined-up and (above all) patient-centred services could be engendered by greater collaborative working among stakeholders, including users. This idea was aligned with NHS policy on service-user involvement at the time, which made it a duty "to involve and consult patients and members of the public not just when a major change is proposed, but in the ongoing planning of services" (Department of Health 2003 p.1).

These three-year pilots were co-funded by the charity Macmillan Cancer Support and the DoH. Mirroring academic definitions (Chadderton 1995, Charles and DeMaio 1993), they were to involve "partnership working," defined by Macmillan as

"where patients, carers and health professionals work collaboratively to bring about tangible service improvements." (Macmillan 2005 p.4)

Two of the seven pilots set up separate service-user forums in response to this brief. The other five included users in their regular multi-disciplinary team, project-management and/or steeringgroup meetings, and it is on these five pilots-where, explicitly or implicitly, users were incorporated into wider professional and managerial teams- that our paper focuses. 
This is an electronic version of an article published in Sociology of Health \& IIIness 33(7): 1050-1065.

The definitive version is available at www.blackwell-synergy.com. (C) 2011 the authors

\begin{tabular}{|l|cccccc}
\hline Site & Users & Physicians & $\begin{array}{c}\text { Interviews } \\
\text { Nurses }\end{array}$ & $\begin{array}{c}\text { Genetic } \\
\text { counsellors }\end{array}$ & Managers & Observations \\
\cline { 2 - 7 } 'Anyton' & 2 & 3 & 2 & 1 & 1 & 1 \\
'Beeville' & 3 & 0 & 2 & 0 & 0 & 2 \\
'Curevale' & 2 & 0 & 5 & 1 & 1 & 3 \\
'Dovering' & 2 & 3 & 2 & 1 & 1 & 4 \\
'Excepton' & 3 & 1 & 2 & 0 & 4 & 9 \\
National level & 4 & 0 & 0 & 0 & 5 & 18 \\
Other sites & 3 & 0 & 1 & 0 & 1 & 2 \\
Total & 19 & 7 & 14 & 3 & 13 & 39 \\
\hline
\end{tabular}

Table 1: Overview of fieldwork activities in each site

The research reported here was conducted as part of a wider evaluation of pilot NHS genetics services. ${ }^{1}$ It took a case-study based approach, designed to facilitate comparison of how the contrasting contexts and approaches of sites involved gave rise to different processes and outcomes (cf. Eisenhardt 1989), and used a range of qualitative methods. Fieldwork involved indepth interviews with 12 involved users (seven interviewed twice), 32 pilot staff (physicians, nurses, genetic counsellors and managers) and five officials from Macmillan, and observational work at 21 site-level meetings (around 35 hours) involving users and professionals (plus 19 national-level meetings, which are not reported in this paper) (see table 1). Interviews lasted between 40 minutes and three-and-a-half hours, and were audio-recorded and fully transcribed. They included questions about various aspects of the user-involvement process, including rationale for involvement, focus of involvement, and how users were influencing the projects. Observational work focused on the interactions between service users and other individuals present at meetings, with attention paid to how both formal structures of the meetings (e.g. agenda items) and informal processes (e.g. behaviour of staff and users) contributed to how users were, and were not, involved. Together, the interview and observational data provided complementary understandings of the way in which service users and staff felt user involvement should operate, and the extent to which this was reflected in practice.

Analysis took a form which sought to combine the merits of locating meaning inductively in the data, while being informed by key issues (sensitising concepts) known to be important from the relevant literatures. GPM led the analysis, reading each data source several times, then (with the assistance of NVivo) generating codes iteratively, guided by both issues identified in the user-involvement literature, and features of the data that became apparent more inductively. These were then grouped into categories that cut across the data, rather than being limited to individual data sources, which were analysed further. Subsequently, we reconsidered these analytical themes and the data they contained in the light of the sociological literature on team work specifically, which was not specifically drawn on ahead of the original analysis. The findings presented below draw on this process, in which the data and their original analysis were revisited and reconsidered in terms of the somewhat different perspective afforded by the team-work literature.

\section{Findings}

Findings are presented in two subsections. First, we cover the way in which user involvement was put into practice in teams in four of the five sites ('Anyton', 'Beeville', 'Curevale' and 'Dovering'). Various macro-level issues across sites impeded the development of an equitable team in which all parties felt valued, and in these four sites, these were exacerbated by micro-level factors. We

\footnotetext{
${ }^{1}$ Ethical approval for this project was obtained from the Trent Multi-centre Research Ethics Committee.
} 
focus on the case of Anyton to exemplify how these issues led to team fragmentation. Then, we contrast this situation with the way in which involvement was developed in the fifth site ('Excepton'). This illustrates how micro-level differences of context and approach seemed crucial in determining how far the ideals of a collaborative team that included users were realised-but this integration in Excepton brought with it challenges to the distinctiveness of users' contribution.

\section{The predominant situation: collaboration frustrated}

The situation in four of the sites, then, was one in which users felt they were not effectively incorporated into the wider professional teams running their pilots; frequently staff too acknowledged that they had fallen short of the equitable partnership they had been asked to create. This could be understood in terms of both macro-level aspects of the pilot programme and wider policy context which affected all five sites, and micro-level organisational set-ups in these four which made it difficult for staff and users to interact and develop trust which might mitigate these challenges.

Macro-level factors. The pilot sites were exhorted by the DoH and Macmillan as sponsors to ensure that service-user involvement was central to their work. However, while Macmillan offered overviews, such as that quoted on page 4, of what involvement should entail, the sponsors provided little specification of how to involve users in their work in a collaborative way, and how pilots' efforts at putting involvement into practice would be evaluated. The exact role for user involvement, and whose interests users were to represent, was never clearly defined. Pilot staff frequently bemoaned the lack of guidance and direction from above about how to 'do involvement', and commented on the difficulties they found in integrating service users into the team. Furthermore, this lack of specification of the means and aims of user involvement was in stark contrast to other aspects of their work. Measures of success such as patient throughput were more actively performance managed, and would be much more important in securing ongoing funding following the pilot period. Consequently, many staff acknowledged that the way in which they set up and pursued user involvement fell short of ideal, because of both competing pressures and this uncertainty about the process and purpose of involvement:

"We were happy that we had a user representative and we ticked that box, which it shouldn't be at all, it should be an active, a really big part of the project." (Nurse, Dovering) $)^{2}$

"[We've done] very little. [...] I don't know how to involve them, I don't know what to ask them to do and it's very difficult getting meetings between us. [The users] just come to the steering-group meetings. But [one user] works, so she can't make them. [Another user] comes. [...] Which I think's very good of her when, really, she must wonder why she comes. Because we're not making full use of her." (Nurse 1, Beeville)

The result was the inclusion of one or two users in each site's team meetings, but often little more than this. At the meetings we attended in these four sites, users were largely present but silent, speaking only during agenda items devoted specifically to user involvement or in response to questions about specific issues. At one (three-hour) meeting in Dovering, for example, users were silent for almost the entire meeting, save for one user commenting briefly on a development event she had attended. Users' contribution to the breadth of strategic issues (of the sort that Macmillan and government guidance on the purpose of involvement highlighted) was thus very limited.

Besides specific ambiguity about the means and end of user involvement, wider forces seemed to orient staff inwards, towards their own professional preoccupations, rather than outwards, towards the kinds of contribution that users did occasionally make. As noted above, staff responsible for pilots had a clear brief-generate patient throughput to demonstrate clinical effectiveness and thus maximise likelihood of ongoing, post-pilot funding-and this to a

\footnotetext{
${ }^{2}$ All primary data excerpts in this paper come from interviews with the parties quoted.
} 
significant extent had to govern their behaviour. The concerns of users were more diffuse. In Anyton, for example, a user described how she had attempted to persuade staff on her hospitalbased pilot of the need to carry out extensive educational work among primary-care clinicians, and evaluate the effectiveness of this training. She found, though, that her pleas fell largely on deaf ears:

"I would have thought that one of the main points, apart from getting however many thousand through the 'sausage machine', was to raise awareness, really build a foundation of understanding within the health community, among district nurses, lead nurses. So I was expecting-I mean I've obviously got it wrong-but I was expecting it to be more getting into [GP] surgeries, because that's what our lot need." (User 1, Anyton)

The pilot lead in Anyton (a clinical geneticist), however, had a very different view of the purpose of the limited educational work included in the project:

"[User and I] have a fundamental disagreement about what the role of that teaching was. $[\ldots]$ The project was set up with a programme of education to raise awareness about cancer genetics, about risk assessment and about the service. So you could argue that we weren't really teaching but advertising, but in a rather detailed way. I've never gone back to get feedback to say, "Was that helpful? Would you like more in the future?" [User] feels that I really should. I haven't had the time to do it; I didn't see that as a priority."

Where the more vociferous users did start to make contributions to the teams in which they were involved, then, they often found their views disregarded:

"I don't think they really understood what we could have given them. Because I think they think we are patients. [...] It's just a strange concept to them, using users. Because I've been used as a user for other things, and it just seems strange that they weren't using us as much as they could have done. I felt a bit of a token gesture." (User 1, Curevale)

The roles of staff, then, were structured by the external priorities placed on pilots, and by their more general positioning within the professional and organisational division of health-care labour, resulting in teams that reproduced these wider structures, and embodied hierarchies that marginalised users.

Micro-level factors. The literature also notes that sometimes, the way in which teams are operationalised can mitigate these challenges, through, for example, nurturing the development of trust (Finn et al., 2010b, Mueller et al. 2000). In these four sites, however, the opposite seemed true: the local organisational context produced clear (social, spatial and temporal) boundaries between staff and users that compounded the factors noted above. The lack of clear roles for users meant that in most sites, their recruitment was an expedient process, with anyone willing and available 'adopted' as a user. In contrast to the process of staff recruitment, users were not judged according to any formal criteria about their skills and competencies. Consequently, when users sought to contribute to pilots, staff were cautious or even, according to some users, suspicious, in the absence of any process by which to assess users' appropriateness to become team members.

This absence of formal vetting at the start of the relationship was followed by a lack of informal opportunities to foster the relationships of trust and reciprocity implied by partnership and team working. While staff employed on the pilots had frequent opportunities to interact with one another in their day-to-day work, in most sites the main point at which they came into contact with users was at steering- or project-group meetings, often as much as three months apart. These meetings were formal affairs, governed by tight agenda and frequently involving not only pilot staff and users, but also wider stakeholders, such as Macmillan representatives, or senior managers from the primary care trust (PCT) or hospital. Consequently, users found themselves trying to foster trusting relationships with staff at meetings where formality precluded the building of much trust, and trying to argue for more expansive, wideranging remits at precisely the points at which staff were most focused on narrow concerns of budget, throughput and sustainability. If there was an opportunity to build relationships that might open the minds of 
staff to partnership — and open the remits of projects to more wideranging ambitions - this was not it.

In these kinds of ways, institutional impediments to a more team-based form of involvement were reinforced by its pilot-level organisation, which bolstered the division between those in the 'team'-staff-and those who were outsiders-the users (and other wider stakeholders, such as senior managers, who could generally, though, rely on positional power for influence). The team itself may not have been characterised by accord and mutuality, but it did at least benefit from ongoing contact that enabled its members to get to know each other and build a sense of commonality and purpose. The performance focus of staff did not encourage them to enter partnerships with individuals they hardly knew, who wished to influence pilots but would not be held accountable for their success or failure, and whose contributions were out of kilter with the main agenda of the meetings they attended. Thus the (micro-level) absence of the opportunity to develop the 'social' aspects of team working - trust, reciprocity, a consistent working relationship built through time-combined with wider (macro-level) forces to preclude the emergence of the 'functional' aspects of team working: shared decision-making processes within pilots.

The situation in Anyton exemplified these problems. Here, the user involved most regularly in the project recounted her growing disappointment at the fact that the 'team' she thought she had joined was not as harmonious as it had first appeared. She described her realisation that even among the project's staff, there were professional hierarchies which she had not expected, as well as inter-personal fractures between some of the pilot's employees. She, meanwhile, found herself on the margins even of this dysfunctional team, as an 'unknown quantity' to staff:

"Their idea of partnership I find quite disturbing, because you've instantly got their hierarchies round the table, between themselves, let alone when the service user turns up. So you've got this consultant thing, and you get your senior nurses, and then you get some sort of also-ran, and some sort of administrative person, and then lower than that is this member of the public that they've had to tick a box to say they had. So I've never felt that they've really come to grips with partnership within their own team, let alone with a member of the public."

Through time, the user found that the pilot's aims were defined by the lead's eagerness to secure as large a throughput of patients as possible. Her own efforts to broaden the remit of the project were unsuccessful, and she felt that even insofar as a team existed, she was indubitably not part of it.

Intriguingly, the Anyton pilot lead and the user deployed the same analogy to describe their experiences of trying to gain legitimacy and influence in the project and in wider NHS cancer provision, comparing it with their experiences of moving into small villages. Their accounts diverged tellingly in the outcomes they describe:

"You make yourself available for talks and things, and you bump into people in the corridor, and you develop a professional relationship where it's friendly. So now I can speak to [breast surgeon] up at [neighbouring hospital] on a friendly basis, whereas before it was senior surgeon, junior consultant. [...] So in some ways it's like moving into a small village and fitting in. And this is exactly what I've done here." (Lead)

"I've lived in this village long enough to know that in a feudal village, you've got a very strange mix of folk, from very different backgrounds, and you can easily offend localsreal locals - by being the incomer. [...] You don't start making pronouncements about farming or sheep prices round here! So really it's just having learnt to be circumspect." (User 1)

Making friends, influencing people and becoming a 'local' in the NHS 'village', then, was a rather different prospect for a consultant compared to a user.

The user acknowledged, though, that the suspicion she felt she had encountered from Anyton's staff was a natural reaction to the inclusion of an individual about whom they knew 
little, and with whom they had few opportunities to form interpersonal bonds or establish common ground. Eventually—and through, by her account, dogged determination-this user felt that she did succeed in gaining a modicum of legitimacy among the staff, though long after Anyton's other user had given up, and only at the very end of the pilot period:

"There was a real move forward. I felt that there was trust. That was the word that I kept on thinking. There was a lot of holding back-I mean, I don't blame them. They don't know who these service users are. They don't know how trustworthy they are. I mean they might be off to the local paper, and spill all sorts of dreadful beans, mightn't they? So I felt that in a way, they'd been right to be cautious. And now they knew it was OK. So I felt that we had a professional kind of relationship, come the end."

\section{Excepton: partnership achieved}

In four sites out of five, the situation described in relation to Anyton was the prevailing pattern, with the local-level social, spatial and temporal organisation of pilots reinforcing wider obstacles that precluded the development of teams that included users. In Excepton, however, things were different. Users and staff alike saw their work as a genuine partnership, as did Macmillan staff:

"They're as much a part of that project as any of the health professionals working on it. It's absolutely true partnership working. And I have to say, as a model it's absolutely outstanding, it's worked exceptionally well. The other projects haven't been quite as successful in that respect." (Macmillan officer)

Certain differences between the set-up in Excepton and the other sites seemed to assist this achievement. For example, where recruitment elsewhere was characterised primarily by expediency, in Excepton the two users were selected purposively by the lead nurse from her past patients on account of their personal characteristics and skills. From the start, then, the users here were far less 'outsiders' than elsewhere. Spatial and temporal organisation consolidated this: project-group meetings were frequent, with the core group of practitioners and users coming together once a month, and at times more frequently to deal with specific issues. It also seemed that the relatively small size of the team and the frequency with which it met rendered it less hierarchically governed than in most other sites, and this meant it was well suited to integrating the users. On an inter-personal level, it was clear that users and staff were both keen to form close, informal bonds that might support their work together. Consequently, they seemed more familiar with one another even than the core staff in some sites:

"It's great, it really works out well because when we've gone to London, we stayed over last time, and we all went out, and there were no barriers at all. [...] It's just such an easy atmosphere, and you feel that you can totally participate, you don't feel you have to think, 'Ooh, should I be commenting like that?"' (User 1)

That the purposive recruitment process had allowed staff to involve the 'right kind' of users, with appropriate attitudes and skills, was certainly crucial to this inter-personal openness and apparent partnership. The service manager commented on the importance of careful selection, to avoid recruiting someone "with a gripe because they've had a raw deal in the service [and to ensure that] it isn't a way of them sabotaging what you're wanting to do." Similarly, the lead nurse stressed the importance of her prior familiarity with the users: "I knew they would be on the same wavelength as me, so that was going to make my life easier than somebody who's very difficult." This undoubtedly helped the flow of the project-group meetings, which were characterised by a consensual decision-making process and often-lengthy conversations about the matters in hand, followed by apparent agreement across the team about the way forward.

An initial congruence of visions, then, was complemented by trust and a conscious effort to ensure that users were actively involved in meetings - in direct contrast to the situation in Anyton and elsewhere. Through time, however, as the pilot moved from working out its purpose, remit and how to achieve these into a more settled phase of service delivery, the role for users became more constrained. The commitment of the staff to involving users remained-indeed 
they actively sought to recruit further users-but the scope for users' involvement now seemed more limited. With the remit of the project established through the consensus of the earlier project-group meetings, the need now was for users to contribute what they could to its success. Effectively, then, users were to draw on their particular skills, alongside professional staff drawing on theirs, to assist progress towards two agreed goals: maximum possible throughput and ongoing funding. To these ends, the users contributed to publicity events for the pilot, and also attended meetings with commissioners alongside pilot staff to signal the commitment of the pilot to genuine user involvement, and demonstrate their own enthusiasm about its work.

Midway through the pilot period, the users were granted 'volunteer status' by Excepton PCT, the pilot's host organisation. Apart from allowing them to make expenses claims, this development also represented an interesting formalisation of their roles and responsibilities:

"It's very much like when you're employed, there's a job description, and you have the discussion with your supervisor or whatever to make sure that you're not having to do things you shouldn't be doing, that you're comfortable with what you're doing, and it looks at us from that point of view to make sure that we're handling things OK. It does the real supervisor bit." (User 1)

This contractual formality - accompanied, as we have seen, by inter-personal informality in the relationships between users and staff in Excepton-reflected the increasingly professionalised role that the users were performing. After receiving ongoing funding from the PCT, staff began working to expand the service to cover neighbouring areas. The service's manager saw an important ongoing role for the users in this expansion process, giving more presentations in nearby towns to which the service was being extended. As the service manager explained, volunteer status meant that users' contribution to this could now be recognised and rewarded:

"They'll get the travel expenses and the food and that sort of thing, but we also pay them something if they're speaking at the events. It's more of a talk, and it's not the full consultancy rate or anything like that! But we try to say 'thank you' in some way. I must admit, at one point it was as if they were full time like we were in the pilot, because they were so committed."

Through time, then, as Excepton's purpose and processes became settled, the role for involvement became more narrowly defined by the needs of the project, in terms of sustainability, throughput and expansion. From the start, these areas had been a crucial focus of the input of users chosen in part for the complementarity of their skills. Attaining volunteer status, though, seemed to reflect an evolution whereby their skills became the defining characteristic of the users' roles. As a Macmillan officer commented, Excepton's users were "not seen as users any longer: they're part of the team." This innocuous observation, however, seemed to highlight a tension in the achievement of 'partnership' in Excepton, where an alignment of interests and a social integration of involvement seemed somehow to precipitate the loss of the distinctive identity and contribution of the users. The existence of the social and structural prerequisites for team work interacted with the emergence, through time, of a stable model of service delivery to give rise to a significant professionalisation of the role, extending to a formalisation of remit (a quasi-job description) and the payment of honoraria. To this extent, the successful integration of users in Excepton into a functional and non-hierarchical team seemed to threaten the distinctiveness of the role of users in the pilot's management, as non-professional representatives of the service-user interest.

\section{Discussion}

The situation in four of the five sites in which user involvement was integrated into the pilots illustrates how many of the challenges which have been shown to impede the realisation of team work across professions also apply to efforts to incorporate users into these teams. External pressures, such as performance requirements, oriented staff away from collaboration with users towards their own priorities; professional norms, values and culture seemed also to disincline 
staff from including users in decision-making processes. The operationalisation of partnership in these sites, then, tended towards conflict and fragmentation, rather than integration between users and the various professional groups present, as found in much of the literature on team working across professions (Dingwall 1980, Donnellon 1996, Finn, 2008, Griffiths 1997). Moreover, many issues identified by the sociological literature on team work were amplified in these sites. Differences of status, culture and socialisation seemed even greater between health professionals and service users than between professions. Furthermore the local social, spatial and temporal organisation of the pilots precluded the opportunity to bridge these gaps. When the only contact between users and the rest of the 'team' was formal, business-focused meetings, often several months apart, users found themselves unable to become trusted, familiar faces and valued team members. Being embedded in the social field of the organisation would seem a vital precondition for this. Thus even if, as the NHS Next Stage Review claims, key interactions and decisions now take place "in multi-disciplinary teams rather than in corridors" (Secretary of State for Health 2008 p.48), being around those corridors is still crucial—as emphasised by the contrasting experiences of getting by in the 'NHS village' described by Anyton's lead and user.

To this extent, the insights suggested by drawing on the organisational sociological literature on team work reinforce and extend some of the findings common from the wider userinvolvement literature internationally. The institutional impediments to the influence of user involvement (Martin 2008b, Barnes et al. 2007, Contandriopoulos 2004) are not unique to public participation, since they also undermine efforts to create collaborative, team-based working within and across health-care professions. However, these impediments do seem more profound in relation to user involvement, due both to the size of the gap between professionals ensconced in the norms, rules and rituals of the health service and users outside these, and due to the way in which micro-level factors reinforce this gap. Given the time and effort needed to build integrated, trusting teams, the way in which the social, spatial and temporal organisation of pilots bolstered the initial disinclination of pilot staff to engage with users seems an important practical issue to address, if we are to move beyond the persistent problems of professional indifference to, or even subversion of, users' views (Harrison and Mort 1998, Sleath and Rucker 2001).

The specific literature on trust and collaborative work is also analytically helpful here, in adding to our understanding of the dynamics of user involvement through team-based organisational forms. Newell and Swan (2000 p.1295) differentiate between three types of trust which they see as underlying the propensity to collaborate and share knowledge:

- companion trust, "based on judgements of goodwill or personal friendships [and] continuing, reciprocal exchange";

- competence trust, "based on perceptions of the others' competence to carry out the tasks that need to be performed," and deriving either from witnessing this competence firsthand or from "contextual cues such as the reputation of the institution that the person works for or the status of the professional group to which they belong"; and

- commitment trust, based on "contractual commitments between the parties," which can act as a more control-based failsafe (cf. Das and Teng 2001) that will continue to facilitate the collaborative partnership if the other two forms of trust are lacking.

The important point here in relation to the development of partnership and team working between professionals and service users is the difficulty of nurturing any of these forms of trust to a significant extent. Companion trust was hard to develop given social, spatial and temporal barriers between users and staff. Competence trust is a problematic concept when the rationale for the inclusion of users is not their expertise but their laity (Martin 2008a): users have no contextual cues to rely on, such as institutional reputation or professional status, and here there was also an absence of clear aims for involvement against which the performance of users might be judged. Commitment trust, too, is a difficult notion in this context: no formal contract existed between users and staff, though given the expectation of Macmillan and the DoH that all pilots develop some form of user-involvement partnership in their projects, there was arguably at least 
a minimum level of commitment from staff to users. This, however, was not necessarily reciprocated: users had no contractual commitment to the projects in which they were involved, and could, if they wanted, walk away without material or symbolic detriment. Nevertheless, this was the form of trust on which the partnerships in four of the sites had to rely in the absence of companion or competence trust. This analysis reinforces the need for social, spatial and temporal organisation of teams that ensures opportunities for companion trust to flourish and to develop competence trust, if efforts to incorporate 'outsiders' such as involved service users into collaborative teams are to be successful. We saw in the example of Anyton that companion and competence trust, according to the user, did eventually develop, but only very late in the threeyear pilot, and after many abortive attempts by the user to exert influence in the team. Difficulties with the development of trust are not unique to users (as Walker et al. 2007 point out, other groups external to strong institutional contexts may face similar challenges), but the problems with developing contractual, competency and companion trust seem especially acute for this group.

Yet in Excepton, things were somewhat different. The micro-level factors that assisted the formation of a more functional partnership were largely absent from the other four sites: a purposive and selective recruitment process; users who were already familiar to some of the staff ahead of the project; frequent meetings involving (only) core staff and users, and other opportunities for bonding among team members; a clear and agreed purpose for user involvement. Companion trust was already in place; competence trust (of a particular kind relevant to the project) was accounted for in the selection of these users as ones who would contribute productively and would not be "difficult," as the nurse here put it. This serves to underline the need to build companion and competence trust to facilitate better collaboration (Newell and Swan 2000)—especially where commitment trust is no more than minimal-by creating and using opportunities for social interaction (Finn et al., 2010b).

However, this 'strategic' approach also seemed to lead to a loss of the distinctiveness of the function of service-user involvement. Following the initial, calculated choice of users to involve, through time the distinction between the contributions of professional staff and users seemed to be diminished, as the users were valued increasingly in the professionalised terms of their skilled contribution to the ongoing success of the service. What this suggests, perhaps, is that users face a particular challenge in the integration-specialisation paradox that team work seeks to address (Donnellon 1996), which does not affect professionals in the same way. Lacking the identity and skill base that define a profession, and which provide a basis for the distinctiveness of its contribution to a team, users seem especially vulnerable to a process of role blurring which can shift the practice of involvement away from the rationale for involving them in the first place. Defined by their laity rather than their specialist knowledge, the role of users can thus become distorted. This suggests that rather than relying on abstract rationales for user involvement relating (for example) to democratic accountability or 'lay expertise' (Martin 2008a), there is a need for a much clearer exposition of what these rationales might mean in practice. Otherwise, the successful functional integration of participatory processes into public-service governance ironically risks bringing with it the unintended consequence of undermining the very reason for doing it.

The notion of the 'team', then - and the emphasis on integration, interdependence and collaborative working that comes with it-represents a model for the incorporation of users into health-service governance that seems laudable-even unopposable. Yet the challenges of making this aspiration real given group interests and powerful institutional forces are profound. These challenges can be overcome so that 'team work' is realised, and users are "not seen as users any longer: they're part of the team," as the Macmillan officer said of Excepton's pilot. However, this empirical analysis shows that in such situations, this apparently positive outcome can obscure a more complex, and less unambiguously desirable, reality. 
This is an electronic version of an article published in Sociology of Health \& IIIness 33(7): 1050-1065. The definitive version is available at www.blackwell-synergy.com. (C) 2011 the authors

\section{Conclusion}

Using concepts from organizational sociological writing on team work which has not previously been applied to this field, we have illustrated how efforts to make teams of professionals and users face similar challenges to those involved in multidisciplinary team work. This highlights difficulties with calls from policymakers for collaborative organisational forms that incorporate those from beyond the NHS in NHS decision-making processes; indeed, due to their location outside the health professions, and the social, spatial and temporal organisation of their inclusion, service users face particular extra difficulties in forging the relationships of trust that form the foundation for collaboration. In this way, by going beyond studying teams that involve only professionals or professional and non-professional staff, our analysis extends both the literature on the challenges involved in realising the team, and that which focuses on user involvement in public-service provision.

Drawing attention to an outlying case where team work was more successful, this paper also indicates some of the interventions that might facilitate more inclusive, collaborative, teamlike partnerships. However, we have sought to show how the particular situation of users renders them vulnerable to a loss of the distinctiveness of their role and contribution, perhaps more so than professional groups attempting to reconcile integration and specialisation. The successful realisation of the team risks sacrificing the distinctiveness of the user role; more attention is thus required to the question of how to operationalise the overarching rationales for involvement in definitions of the role of the service user qua service user, to provide a robust basis for retaining the distinctiveness of this role in integrated teams and partnerships.

\section{Acknowledgements}

We are grateful to Celia Davies, Mary Dixon-Woods, Sue Newell and two anonymous referees for critical comments which helped us to improve this paper. We would also like to thank those who participated in this study, and the funders (Department of Health and the Centre for Social Research in Health and Health Care, University of Nottingham).

\section{References}

Abelson,J., Forest,P., Eyles,J., Smith,P., Martin,E. and Gauvin,F. (2003) Deliberations about deliberative methods: issues in the design and evaluation of public participation processes, Social Science \& Medicine, 57,2,239-251.

Barnes,M., Newman,J. and Sullivan,H. (2007) Power, participation and political renewal. Bristol: Policy Press.

BRI Inquiry (2001) Learning from Bristol. London: HMSO.

Chadderton,H. (1995) An analysis of the concept of participation within the context of health care planning, Journal of Nursing Management, 3,5,221-228.

Charles,C., and DeMaio,S. (1993) Lay participation in health care decision making: a conceptual framework, Journal of Health Politics, Policy and Law, 18,4,881-904.

Contandriopoulos,D. (2004) A sociological perspective on public participation in health care, Social Science \&o Medicine, 58,2,321-330.

Cott,C. (1998) Structure and meaning in multidisciplinary teamwork, Sociology of Health \& Illness, 20,6,848-873.

Das,T.K. and Teng,B. (2001) Trust, control, and risk in strategic alliances: an integrated framework, Organization Studies, 22,2,251 -283.

Department of Health (2003) Strengthening accountability: policy guidance. London: Department of Health.

Dingwall,R. (1980) Problems of teamwork in primary care. In Lonsdale,A., Webb,A. and Briggs,T. (eds)Teamwork in the personal social services and health care. London: Croom Helm, pp.111-137. 
This is an electronic version of an article published in Sociology of Health \& IIIness 33(7): 1050-1065. The definitive version is available at www.blackwell-synergy.com. (C) 2011 the authors

Donnellon,A. (1996) Team talk. Boston: Harvard Business School Press.

Eisenhardt,K.M. (1989) Building theories from case study research, Academy of Management Review, $14,4,532-550$.

Finn,R. (2008) The language of teamwork: reproducing professional divisions in the operating theatre, Human Relations, 61,1,103-130.

Finn,R., Learmonth,M. and Reedy,P. (2010a) Some unintended effects of teamwork in healthcare, Social Science \& Medicine, 70,8,1148-1154.

Finn,R., Currie,G. and Martin,G. (2010b) Team work in context: institutional mediation in the public-service professional bureaucracy, Organization Studies, 31,8,1069-1097.

General Medical Council (2006) Good medical practice. London: GMC.

Glendinning,C. (2003) Breaking down barriers: integrating health and care services for older people in England, Health Policy, 65,2,139-151.

Griffiths, L. (1997) Accomplishing team: teamwork and categorisation in two community mental health teams, Sociological Review, 45,1,59-78.

Harrison,S. and Mort,M. (1998) Which champions, which people? Public and user involvement in health care as a technology of legitimation, Social Policy \& Administration, 32,1,60-70.

Learmonth,M. (2009) 'Girls' working together without 'teams': How to avoid the colonization of management language, Human Relations, 62,12,1887-1906.

Lotz,M. and Kristensen,P.H. (2009) Taking teams seriously in the co-creation of economic agency: towards an organizational sociology of teams. http://project.hkkk.fi/translearn/Publications/090301Teampaper_ML_PHK_MR.pdf.

Macmillan (2005) Working brief 5. London: Macmillan.

Martin,G.P. (2008a) 'Ordinary people only': representativeness, knowledge, and the publics of public participation in healthcare, Sociology of Health \& Illness, 30,1,35-54.

Martin,G.P. (2008b) Representativeness, legitimacy and power in public involvement in healthcare management, Social Science \& Medicine, 67,11,1757-1765.

Martin,G.P., Currie,G. and Finn,R. (2009) Leadership, service reform, and public-service networks: the case of cancer-genetics pilots in the English NHS, Journal of Public Administration Research \& Theory, 19,4,769-794.

Milewa,T., Valentine,J. and Calnan,M. (1999) Community participation and citizenship in British health care planning: narratives of power and involvement in the changing welfare state, Sociology of Health and Illness, 21,4,445-465.

Mueller,F., Proctor,S. and Buchanan,D. (2000) Teamworking in its context(s): Antecedents, nature and dimensions. Human Relations, 53,11,1387-1424.

Newell,S. and Swan,J. (2000) Trust and inter-organizational networking, Human Relations, $\mathbf{5 3 , 1 0 , 1 2 8 7 - 1 3 2 8 .}$

Opie,A. (1997) Thinking teams thinking clients: issues of discourse and representation in the work of health care teams, Sociology of Health \& Illness, 19,3,259-280.

Ring,P.S. and van de Ven,A.H. (1994) Developmental processes of cooperative interorganizational relationships, Academy of Management Review, 19,1,90-118.

Rutter,D., Manley,C., Weaver,T., Crawford,M.J. and Fulop, N. (2004) Patients or partners? Case studies of user involvement in the planning and delivery of adult mental health services in London. Social Science \& Medicine, 58,10,1973-1984.

Secretary of State for Health (2008) High quality care for all. London: The Stationery Office.

Sleath,B. and Rucker,T.D. (2001) Consumer participation in health policy decisions: empowerment or puffery? Journal of Health Care for the Poor and Underserved, 12,1,35-49.

Theberge,N. (2008) The integration of chiropractors into healthcare teams: a case study from sport medicine, Sociology of Health \& Illness, 30,1,19-34.

Tomlinson,F. (2005) Idealistic and pragmatic versions of the discourse of partnership, Organization Studies, 26,8,1169-1188.

Walker,R., Bisset,P. and Adam,J. (2007) Managing risk: Risk perception, trust and control in a 
This is an electronic version of an article published in Sociology of Health \& IIIness 33(7): 1050-1065.

The definitive version is available at www.blackwell-synergy.com. (C) 2011 the authors

Primary Care Partnership, Social Science \& Medicine, 64,4,911-923. 